\title{
Molecularly confirmed Li-Fraumeni-like syndrome in a patient with breast cancer and a low pre-test probability for harboring a germline CHEK2 truncation
}

\author{
Steven Sorscher* \\ Oncology Division, Wake Forest School of Medicine, One Medical Center Blvd, USA
}

\section{Introduction}

Next generation sequencing (NGS) affords patients the potential opportunity to receive highly targeted therapies if actionable genetic abnormalities are identified in their tumors. Currently, nearly all available approved targeted therapies target oncogene products as opposed to replacing deficient tumor suppressor gene (TSG) products. Generally, replacing deficient TSG products in a malignancy is presumed to only be effective if there is near total lack of expression of a particular normal TSG product, as would occur with LOH for the particular TSG.

Molecularly defined, Li-Fraumeni syndrome is due to inheritance of a mutated TP53 [1,2]. Li-Fraumeni syndrome patients are at particularly increased risk of developing breast cancers, brain tumors, leukemia and adrenal cortical cancers [2-4]. Because the normal protein expressed by the TSG checkpoint kinase 2 (CHEK2) activates TP53, lack of expression of CHEK2 is thought to in many ways be tantamount to lack of expression of normal TP53. Therefore, germline alterations in the CHEK2 gene result in what has been described as "Li-Fraumenilike" syndrome and patients would be expected to have increased risk of a variety of cancers [1-4].

Here, I describe a patient whose breast cancer harbored a truncation in CHEK2, c.1100del. The relatively high mutation allelic frequency (MAF) confirmed LOH of CHEK2 as the gatekeeper event in tumorigenesis. The particular CHEK2 alteration and the roughly $50 \%$ frequency of that mutation seen in germline testing confirmed that the patient is of a molecularly diagnosed Li-Fraumeni-like family. Although estimating the risk for Li-Fraumeni-like related cancers when the c.1000del CHEK2 is discovered in a patient with a high pretest probability of harboring the truncation is fairly well defined, the penetrance in the described patient (with no clearly significant family history of Li-Fraumeni-like associated cancers and therefore a low pretest probability) is more problematic.

\section{Case report}

CC is a 66 year old woman who underwent a left-sided mastectomy and sentinel lymph node evaluation for a T2NO (Stage IIA) ER positive, PR positive, HER2 positive (IHC $3+$ ) invasive ductal breast adenocarcinoma in September 2015 and received adjuvant systemic therapy with docetaxel/carboplatin/trastuzumab/pertuzumab and, after completing the chemotherapy, initiated aromatase inhibitor therapy.

Family history $(\mathrm{FH})$ included a maternal aunt with ovarian cancer (age 60), a brother with multiple myeloma (age 64) and a maternal aunt (age 82) and a paternal half-sister (age unknown) with colorectal cancers.

Next generation sequencing (NGS) of her cancer demonstrated a genomic alteration in CHEK2 T367fs ${ }^{\star} 15$ (c.1100del) (Foundation Medicine, Inc, Cambridge, MA 02141). Germline testing showed the same deleterious alteration (Myriad Genetics, Salt Lake City, UT, 84108).

Based on these results, the unreported mutation allelic frequencies (MAF) $=71 \%$ for the breast cancer (Foundation Medicine) and MAF $=49.6 \%$ (Myriad Genetics) were obtained for the CHEK2 alteration.

\section{Discussion}

The CHEK2 TSG product primarily functions to activate TP53 and induce cells to undergo cell-cycle arrest or apotopsis. Lack of expression of functional CHEK2 would be considered a gatekeeper event in transformation to malignancy. Lack of expression can be secondary to inheritance of c.1100del CHEK2 followed by inactivation of expression of wild-type (WT) CHEK2 genes in a normal breast cell or through somatic mutations or inactivation of WT genes in a normal breast cell [2-4]. The later events appear to be far less common in breast cancer. For example, it is estimated that grossly reduced or absent CHEK2 protein expression is seen in only 4 to $14 \%$ of familial breast tumors from CHEK2 non-carriers [5,6]. A study of 10, 860 breast cancer cases and 9,065 controls from 10 case-control studies in 5 countries found a CHEK2 variant in $1.9 \%$ of all cases and $0.7 \%$ of controls. The authors concluded that the 1100 delC allele confers roughly a twofold elevation in breast cancer risk in women unselected for family history [7]. In the $\mathrm{UK}$, the absolute cumulative risk of breast cancer among 1100 delC allele carriers was roughly $13.7 \%$ by the age of 70 compared to $6.1 \%$ of non-carriers [7].

CC'c age of diagnosis and lack of a FH suggestive of a familial syndrome (see above) would suggest that she is a non-carrier of CHEK2, and therefore not of a Li-Fraumeni-like family. 1100delC CHEK2 germline alterations have been associated particularly with an increased risk of developing breast, prostate and kidney cancers and not with ovarian cancer and apparently not (or minimally) with colorectal cancer risk $[3,8]$. A literature search revealed no studies suggesting an

Correspondence to: Steven Sorscher, Professor of Medicine, Oncology Division, Wake Forest School of Medicine, One Medical Center Blvd, Winston-Salem, NC 27104, USA, Tel: 336-716-0230, E-mail: ssorsche@wakehealth.edu

Received: January 27, 2017; Accepted: February 14, 2017; Published: February 17,2017 
association with multiple myeloma. Also, denovo mutations have very rarely been reported [9].

However, the germline MAF of $49.6 \%$ confirms her to be of a molecularly-defined Li-Fraumeni-like family. (Of note, there is also evidence that germline CHEK2 alterations are associated with HER2 positive disease, as she had [10]). Per accepted guidelines, this diagnosis would place her and other family members harboring the same germline alteration at higher risk for a variety of cancers. . The pre-test probability is the probability of the patient being proven to have the disease (in this case, Li-Fraumeni-like syndrome) before having had the test. In spite of this guideline, her very low pre-test probability of harboring the CHEK2 alteration that testing demonstrated raises the question of whether the penetrance established in other carriers (who have a higher pre-test probability) applies to this somewhat "incidentally" discovered germline alteration carrier. The guidelines suggest that regardless of the $\mathrm{FH}$, screening and even prophylactic measures for carriers of nearly all known cancer-associated genes be the same whether the patient has a strong family history or not [11]. Paradoxically, if a patient with a very high pre-test probability tests negative, it is recommended that the screening approach to that patient be the same had they tested positive for a mutation in the gene of concern [11].

Obtaining the unreported MAF from the tumor is also relevant to the issue of the significance of the germline mutation in this patient and her family as well. The MAF of $71 \%$ would appear to confirm loss of heterozygosity as the mechanism. Had the MAF in the tumor been less than $50 \%$, it would remain possible that inactivation of expression of WT CHEK2 was still the gatekeeper molecular transforming event, but with a lower MAF it would also be possible that the breast cancer was sporadic. Had the cancer been sporadic, this would be further evidence of, for example, linked polymorphisms protecting this $\mathrm{Li}$ Fraumeni-like patient and her family from the cancers associated with the inherited CHEK2 alteration.

Finally, NGS sequencing is typically requested to identify "actionable" molecular abnormalities. In the case of oncogenes, the MAF is likely irrelevant if a targeted agent that inhibits the activity of an expressed oncogene is available. On the other hand, agents that target the lack of expressed normal TSG products presumably would be effective only if the loss of the TSG product is near total in the malignancy. Therefore, if there were a drug known to target the lost TSG CHEK2 product, the CHEK2 MAF of $71 \%$ would suggest that such an agent might be effective.
In summary, obtaining the unreported MAFs for both the identified germline c.1100del CHEK2 and the same CHEK2 alteration identified by NGS molecularly confirmed that this patient is of a Li-Fraumeni-like family, although it is unclear as to whether she and other carriers in her family might expect the same risk of Li-Fraumeni-like associated cancers as Li-Fraumeni-like patients identified who have high pretest probabilities of harboring the CHEK2 germline mutation or meet clinical criteria for Li-Fraumeni-like syndrome. Also, if there were a targeted agent shown to replace lacking CHECK2 normal protein product, the high MAF in the tumor of this patient would increase the probability of efficacy compared to use in a tumor where the known normal TSG CHEK2 product was expressed.

\section{References}

1. Li F, Fraumeni JF (1969) Soft tissue sarcoma, breast cancer, and other neoplasms. A familial syndrome? Ann Intern Med 71: 747-752. [Crossref]

2. Malkin D (2011) Li-fraumeni syndrome. Genes Cancer 2: 475-484.[Crossref]

3. Nevanlinna H, Bartek J (2006) The CHEK2 gene and inherited breast cancer susceptibility. Oncogene 25: 5912-5919.[Crossref]

4. Bell DW, Varley JM, Szydlo TE, Kang DH, Wahrer DC, et al. (1999) Heterozygous germ line hCHK2 mutations in Li-Fraumeni syndrome. Science 286: 2528-2531. [Crossref]

5. Vahteristo P, Bartkova J, Eerola H, Syrjäkoski K, Ojala S,et al. (2002) A CHEK2 genetic variant contributing to a substantial fraction of familial breast cancer. $\mathrm{Am} J$ Hum Genet71: 432-438. [Crossref]

6. Oldenberg RA, Kroeze-Jansema K, Kraan J, Morreau H, Klijn JG, et al. (2003) The CHECK $2 * 1100$ delC variant acts as a breast cancer risk modifier in non-BRCA1/ BRCA2 mulltiple-cancer families. Cancer Res63:8153-8157.[Crossref]

7. TheCHEK 2 Breast Cancer Case-Control Consortium (2004) CHEK $2 * 1100 \mathrm{delC}$ and susceptibility to breast cancer: a collaborative analysis involving 10,860 breast cancer cases and 9,065 controls from 10 studies. Am J Hum Genet 74:1175-1182. [Crossref]

8. Cybulski C, Wokoiorczyk D, Kidny J, Kurzawski G, Suchy J, et al. (2007) Germline CHEK2 mutations and colorectal cancer risk: different effects of missense and truncating mutations? Eur J Hum Genet 15:237-241.[Crossref]

9. Zhuang X, Li Y, Cao H, Wang T, Chen J, et al. (2016) Case report of a Li-Fraumeni syndrome-like phenotype with a de novo mutation in CHEK2. Medicine (Baltimore) 95 : e4251.[Crossref]

10. Ramamurthy C, Shameem R, Forman A, Dolinsky JS, Speare V, et al. (2016). Risk of HER2-positive breast cancer among germline CHEK2 mutation carriers with breast cancer. J Clin Oncol 34.

11. Daly MD, Pilarski R, Berry M (2017) National Comprehensive Cancer Network: Genetic/familial high-risk assessment: breast and ovarian version 2.2017. [https:// www.ncen.org]

Copyright: (C2017 Sorscher S. This is an open-access article distributed under the terms of the Creative Commons Attribution License, which permits unrestricted use, distribution, and reproduction in any medium, provided the original author and source are credited. 